\title{
Analysis of IgG Responses to P. falciparum Antigens MSP1, MSP4-20 and MSP4-40 during Severe Malaria in Dakar, Senegal
}

\section{Bruce Shinga Wembulua ${ }^{*}$, Kalilou Diallo², Folly Mawulolo Gaba ${ }^{3}$, Babacar Mbengue and Louise Fortes $^{1}$}

${ }^{1}$ Department of Infectious and Tropical Diseases, Fann University Hospital, Dakar, Senegal

${ }^{2}$ Infectious and Tropical Diseases Unit, Hôpital De La Paix, Ziguinchor, Senegal

${ }^{3}$ Department of immunology, Cheikh Anta Diop University, Dakar, Senegal

*Corresponding author: Bruce Shinga Wembulua, Department of Infectious and Tropical Diseases, Fann University Hospital, Dakar, Senegal, Tel: 0033751498086

\begin{abstract}
Background: Anti-Merozoite surface proteins (MSP) IgG response are really associeted with lower malaria morbidity and mortality. However, only few studies have looked at the implications of their variations on the course of clinical and biological forms of severe malaria.

Methods: This cross-sectional, prospective, and analytical study evaluates the $\lg G$ responses against the antigens MSP1, MSP4-20 and MSP4-40 of $P$. falciparum during severe malaria in patients hospitalized in the infectious diseases department of Fann university hospital from October 1, 2017, to November 30, 2019.

Results: A total of 86 patients were selected. The average age of patients was $34 \pm 17$ years. Renal $(74.4 \%)$, neurological $(63.9 \%)$ and hepatic $(55.8 \%)$ impairment were the main WHO-forms of severity. The mean hemoglobin level was $11.2 \pm 3 \mathrm{~g} / \mathrm{dL}$ The parasitemia was lower $(<0.5 \%)$ in $80 \%$ of cases. In the evolution, 10 patients deceased making the lathality rate of $11.6 \%$. The presence of associated diagnoses and comorbidities was linked to low levels of anti-MSP4-40 IgG ( $p=0.032)$ and anti-MSP1 ( $p$ $=0.037$ ), respectively. The number of failures per patient increased with the fall in the levels of anti-MSP1 IgG ( $p=$ $0.017)$ and MSP4-20 ( $p=0.04)$. Only high levels of antiMSP4-40 lgG were statistically associated with low lethality $(p=0.037)$.

Conclusion: Anti-MSP1, MSP4-20, and MSP4 antibodies are involved in the anti-plasmodial humoral response in severe malaria. Their blood levels can serve as prognostic biomarkers for an optimal management.
\end{abstract}

\section{Keywords}

$P$. falciparum, Severe malaria, IgG, Merozoite surface protein (MSP), Hospital, Dakar, Senegal

\author{
Abbreviations \\ MSP: Merozoite Surface Antigen; IgG: Immunoglobulin G; \\ OD: Optical Densities; OD rt: Optical Density Ratio
}

\section{Introduction}

Plasmodium falciparum malaria remains a global public health problem. The World Health Organization (WHO) estimates that 3.3 billion people are at risk of infection with an incidence of 229 million cases associated with 409,000 deaths per year [1]. However, a significant decline in endemicity has been noted over the past 20 years [1-5]. In the WHO Africa Region, incident cases fell from 294 cases per 1000 in 2010 to 229 cases in 2018 (-22\%) [5]. In Senegal, between 2018 and 2019 , the incidence of malaria decreased by $35.4 \%$ [6]. This decrease in endemicity exposes to severe forms $[2,7]$. Adequate management of these severe forms implies a better understanding of their pathophysiology in which the involvement of antibodies has been widely demonstrated [8-10]. Several Senegalese studies have confirmed the significant relationship between high levels of anti-MSP1, MSP4-20, MSP4-40 antibodies (Ab) and low malaria morbidity and mortality [11-13]. However, only

Citation: Wembulua BS, Diallo K, Gaba FM, Mbengue B, Fortes L (2022) Analysis of IgG Responses to $P$. falciparum Antigens MSP1, MSP4-20 and MSP4-40 during Severe Malaria in Dakar, Senegal. Int J Trop Dis 5:056. doi.org/10.23937/2643-461X/1710056

Accepted: February 05, 2022; Published: February 07, 2022

Copyright: (C) 2022 Wembulua BS, et al. This is an open-access article distributed under the terms of the Creative Commons Attribution License, which permits unrestricted use, distribution, and reproduction in any medium, provided the original author and source are credited. 
a few have been interested in the implications of their variation on the course of clinical and biological forms of severe malaria $[11,14]$. Thus, our study was conducted to analyze the IgG responses against $P$. falciparum MSP1, MSP4-20 and MSP4-40 antigens during severe malaria in patients hospitalized in the infectious diseases department of Fann university hospital.

\section{Materials and Methods}

\section{Population and study design}

This is a cross-sectional, prospective, and analytical study. All patients presenting severe malaria according to the WHO severity criteria [15] and hospitalized in the infectious diseases department of Fann university hospital from October 1, 2017, to November 30, 2019, were eligible for the study. Informed consent was previously obtained from patients or their representatives, before inclusion. Patients who were infected with HIV and those who had not given their consent were systematically excluded. Data were collected using a standard questionnaire To separate-demographic, clinical, biological, immunological, therapeutic, and evolution characteristics.

\section{Definition of variables}

In this study, chronic pathologies (diabetes, high blood pressure, asthma, etc.) constituting a field on which severe malaria has developed have been considered comorbidities. Acute pathologies (bacterial infection, recent stroke, etc.) intercurrent to severe malaria were considered associated diagnoses. Probable bacterial infection was retained as an associated diagnosis ahead of the persistence of the infectous syndrome syndrome after 3 doses of artesunate and a negative control (thick blood film analysis). In case a bacterium was isolated, the infection was considered confirmed (bacteremia). Clinical, biological and parasitological forms of severe malaria were defined according to the WHO severity criteria [15]. Renal, hematological, electrolyte, and hepatic disturbances were defined according to the table of usual biological constants of the Perlemuter 2017 therapeutic guide [16].

\section{Laboratory methods}

The thick blood films were stained by the MayGrünwald Giemsa at $10 \%$ and read under the microscope using the 100-immersion objective compared to a field of about 200 leukocytes. For the serological study, whole blood samples were taken from the elbow crease with an EDTA tube. The serum of each sample was isolated after centrifugation at 4000 revolutions/min for 5 minutes. The anti-MSP1, MSP4-20 and MSP4-40 IgGs were dosed by the ELISA technique according to the protocol described by Mbengue, et al. [10,12]. The optical densities (OD) measured with a spectrophotometer at $450 \mathrm{~nm}$ (Asys Hitech ${ }^{\circledR}$ ) were used to express the results in OD ratio (ODrt) which corresponds to the ratio of the OD of the sample to that of a systematic negative control (pool of sera of unexposed Europeans). The validity of negative responses was checked with an African naïve control serum. A pool of sera collected from hyperimmune subjects served as a positive control. Were considered as responders, patients showing OD rt of $\geq 2$ (the mean of naïve controls plus 2 standard deviations, representing a positivity of more than $95 \%$ ).

\section{Data analysis}

Data were analyzed using Statview 5.1 software (SAS Institue, Cary, NJ). The comparison of the Ab levels according to the clinical-biological parameters was carried out using the non-parametric Kruskal-Wallis test after verification of the normality of the distribution. The difference was considered significative with a P-value $<0,05$.

\section{Results}

\section{Epidemiological, therapeutic, and evolution characteristics}

A total of 86 patients were selected. The average age was $34 \pm 17$ years. In their history, $31.4 \%$ of patients had some notion of travel to other regions of Senegal in the past 3 months. High blood pressure was the most reported comorbidity $(9.3 \%)$. Half $(54.5 \%)$ of the comorbid patients were over 55 years of age. In addition, another diagnosis was associated with severe malaria in

Table 1: Comorbidity and associated diagnoses of patients hospitalized for severe malaria at the infectious and tropical department of Fann university hospital from October 1, 2017 to November 30, 2019 ( $n=86)$.

\begin{tabular}{|c|c|c|c|}
\hline \multicolumn{2}{|c|}{ Comorbidities and associated diagnoses } & \multirow{2}{*}{\begin{tabular}{|l|}
$f$ \\
8
\end{tabular}} & \multirow{2}{*}{\begin{tabular}{|l|}
$\%$ \\
9.3 \\
\end{tabular}} \\
\hline & Hypertension & & \\
\hline & Smoking & 6 & 7 \\
\hline \multirow[t]{3}{*}{ Comorbidity } & Alcoholism & 4 & 4.7 \\
\hline & Others $^{*}$ & 7 & 8.1 \\
\hline & None & 69 & 80.2 \\
\hline \multirow{3}{*}{ Type of associated diagnostics } & Probable bacterial infections $^{* *}$ & 16 & 80.0 \\
\hline & Bacteremia & 3 & 15.0 \\
\hline & Stoke & 1 & 5.0 \\
\hline
\end{tabular}

*Asthma (2), diabetes (1), epilepsy (1), Myeloma (1), hepatitis B (2); *Encephalitis (8), cholecystitis (3), acute gastroenteritis (4), Nonspecific pneumonia (1) 
20 patients $(23.3 \%), 20 \%$ of whom were over 60 -yearsand hepatic (55.8\%) damage were the main forms of old (Table 1). Renal (74.4\%), neurological (63.9\%) severity. The mean hemoglobin level was $11.2 \pm 3 \mathrm{~g} / \mathrm{dl}$

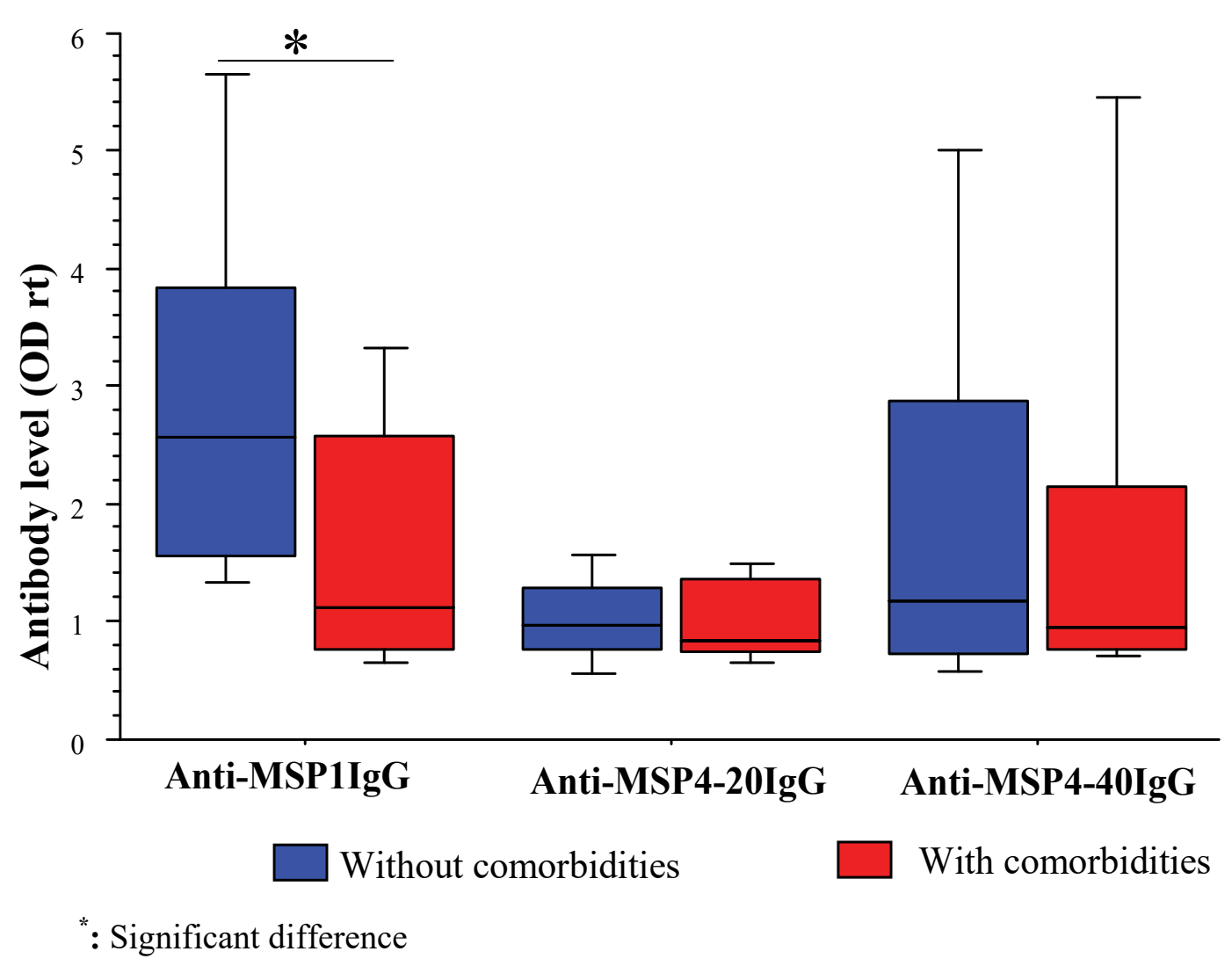

Figure 1: Comparison of anti-MSP1, MSP4-20 and MSP4-40 lgG levels of patients hospitalized for severe malaria at the infectious and tropical department of Fann university hospital, depending on the presence or absence of comorbidities.

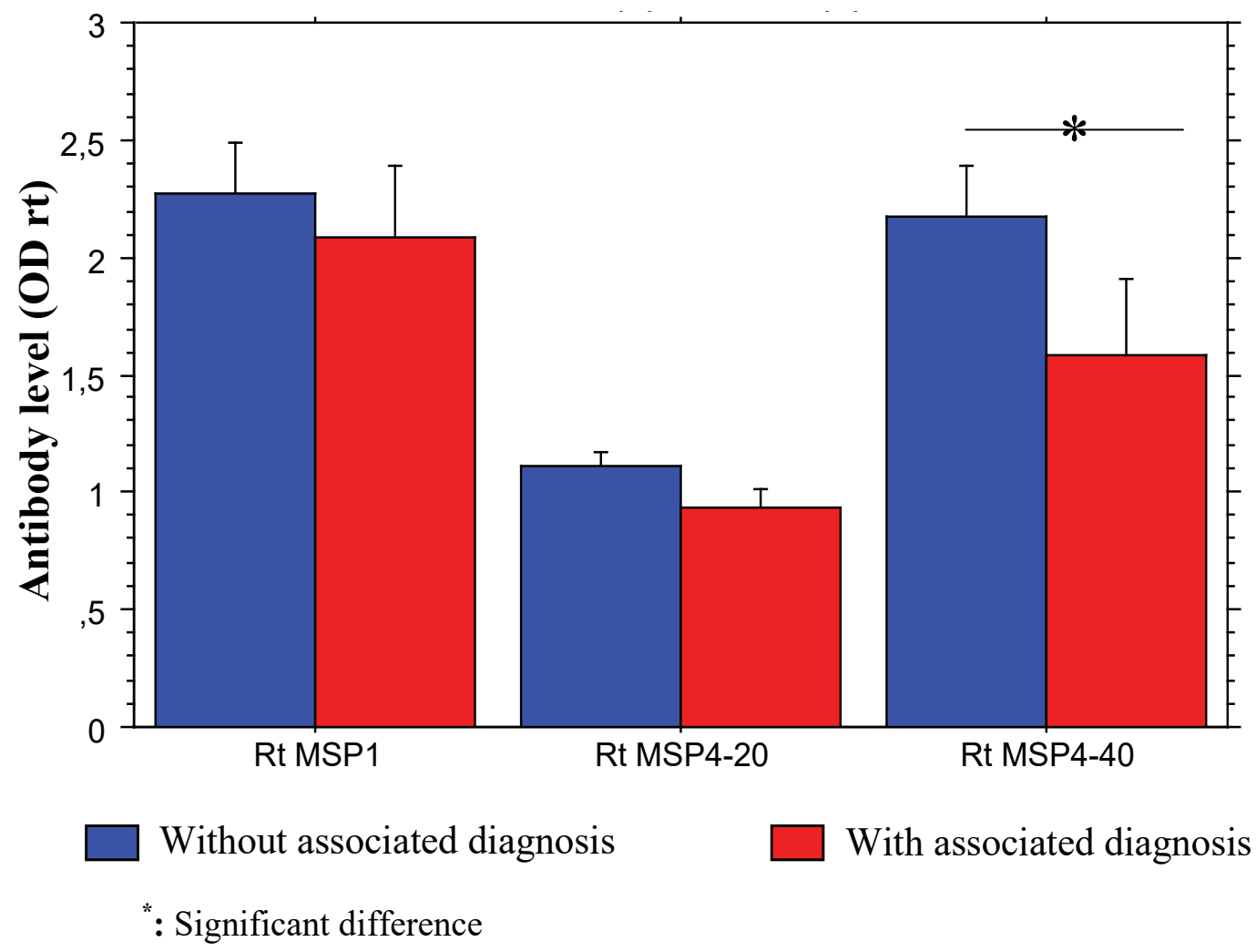

Figure 2: Comparison of anti-MSP1, MSP4-20 and MSP4-40 lgG levels of patients hospitalized for severe malaria at the infectious and tropical department of Fann university hospital, depending on the presence or not of an associated diagnosis. 
with low parasitemia $(<0.5 \%)$ in $80 \%$ of cases parenteral artesunate was the drug of choice in all our patients. The outcome was favorable in 76 patients $(88.4 \%)$. Ten patients had died making a lethality rate of $11.6 \%$.

\section{Analysis of anti-MSP IgG responses}

We noted a significant decrease in the levels of anti-MSP4-40 IgG ( $p=0.032)$ and anti-MSP1 IgG ( $p=$ 0.037 ) in patients presenting an associated diagnosis and a comorbidity, respectively (Figure 1 and Figure 2). Comparing the patients antibody levels with the number of visceral failures, we observed that antiMSP1 ( $p=0.017$ ) and anti-MSP4-20 lgG levels was significantly lower $(p=0.04)$ in patients with higher number of impaired organs (Figure 3). On the other hand, depending on the evolution of the patients, high levels of anti-MSP4-40 IgG were statistically associated with low lethality ( $p=0.037$ ) (Figure 4).

\section{Discussion}

High levels of antibodies to merozoite surface antigens are associated with low malaria morbidity and mortality [11-13]. These antibody responses are, however, heterogeneous and variable depending on several factors. Kwenti TE, et al. [17] in their study in
Cameroonian children, observed that anti-MSP1p19 antibody responses varied according to age and different bioecological strata. Female gender was associated with high levels of anti-MSP1p19 antibodies in a Ugandan study [18]. In Senegal, Mbengue, et al. [13] noted that the association of antibody responses and parasitemia was dependent on anti-MSP3 IgG in children but linked to anti-MSP1p19 IgG in adults. In Malawi, Dobaño C, et al. [19] noted that anti-MSP1 and MSP2 IgG levels varied according to clinical phenotypes. Our study adds to this set of factors, the associated diagnosis, and comorbidities. They were associated with low levels of anti-MSP4-40 and anti-MSP1 IgG, respectively. Our results could explain the increased risk of pernicious attacks in diabetics and obese people described by Wyss $\mathrm{K}$, et al. [20] as well as the high lethality in patients with a combination of malaria and bacterial infection in the series of Diop A, et al. [21]. In addition, the high lethality in subjects over 60 years of age in our series may also be linked to this weak antibody response since more than half $(55.6 \%)$ of them had at least one comorbidity.

It is evident that severe forms of malaria result from a complex cascade of events including quantitative and/ or qualitative defects in protective antibody responses

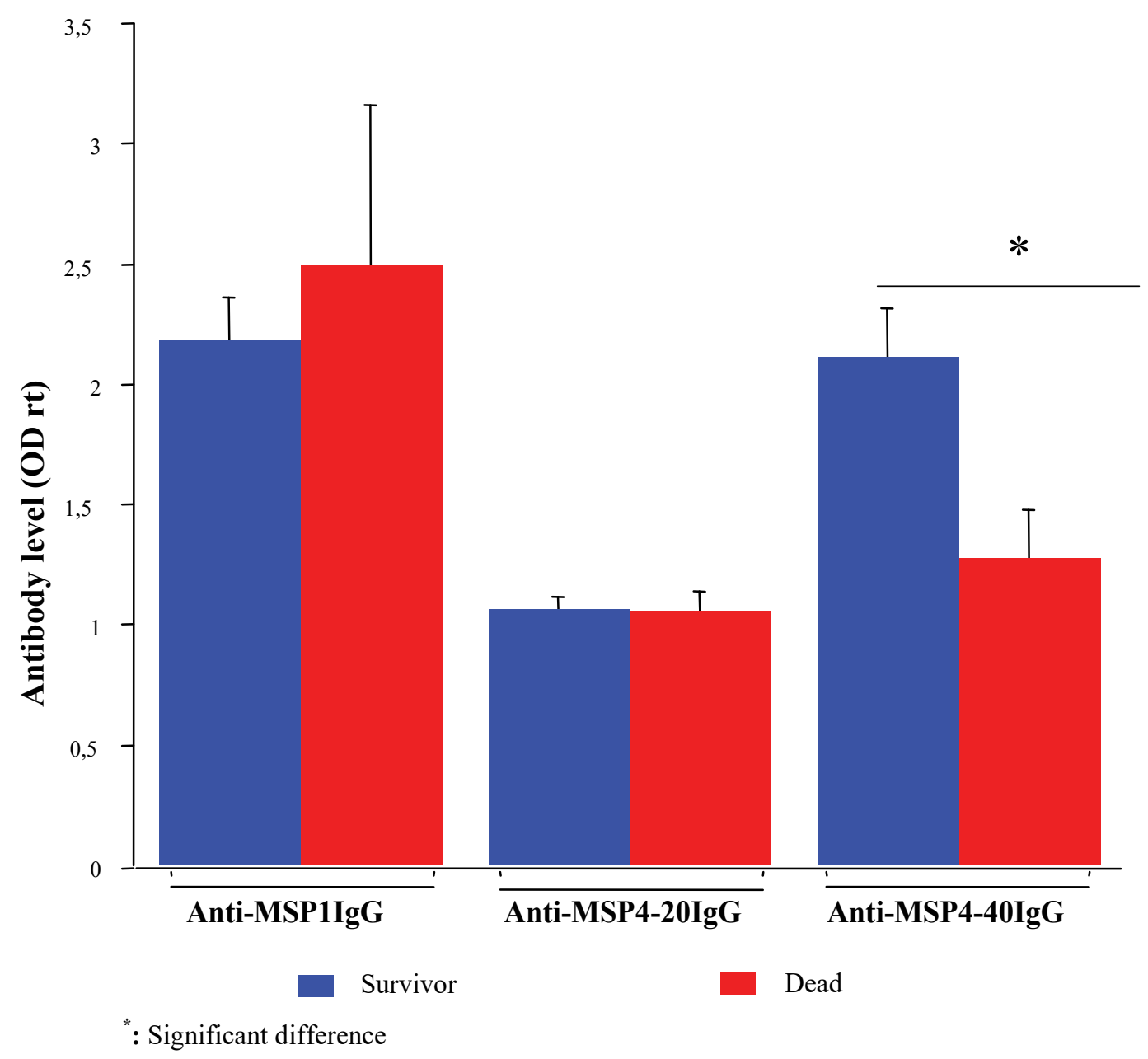

Figure 3: Comparison of anti-MSP1, MSP4-20 and MSP4-40 lgG levels of patients hospitalized for severe malaria at the infectious and tropical department of Fann university hospital, depending on the evolution. 


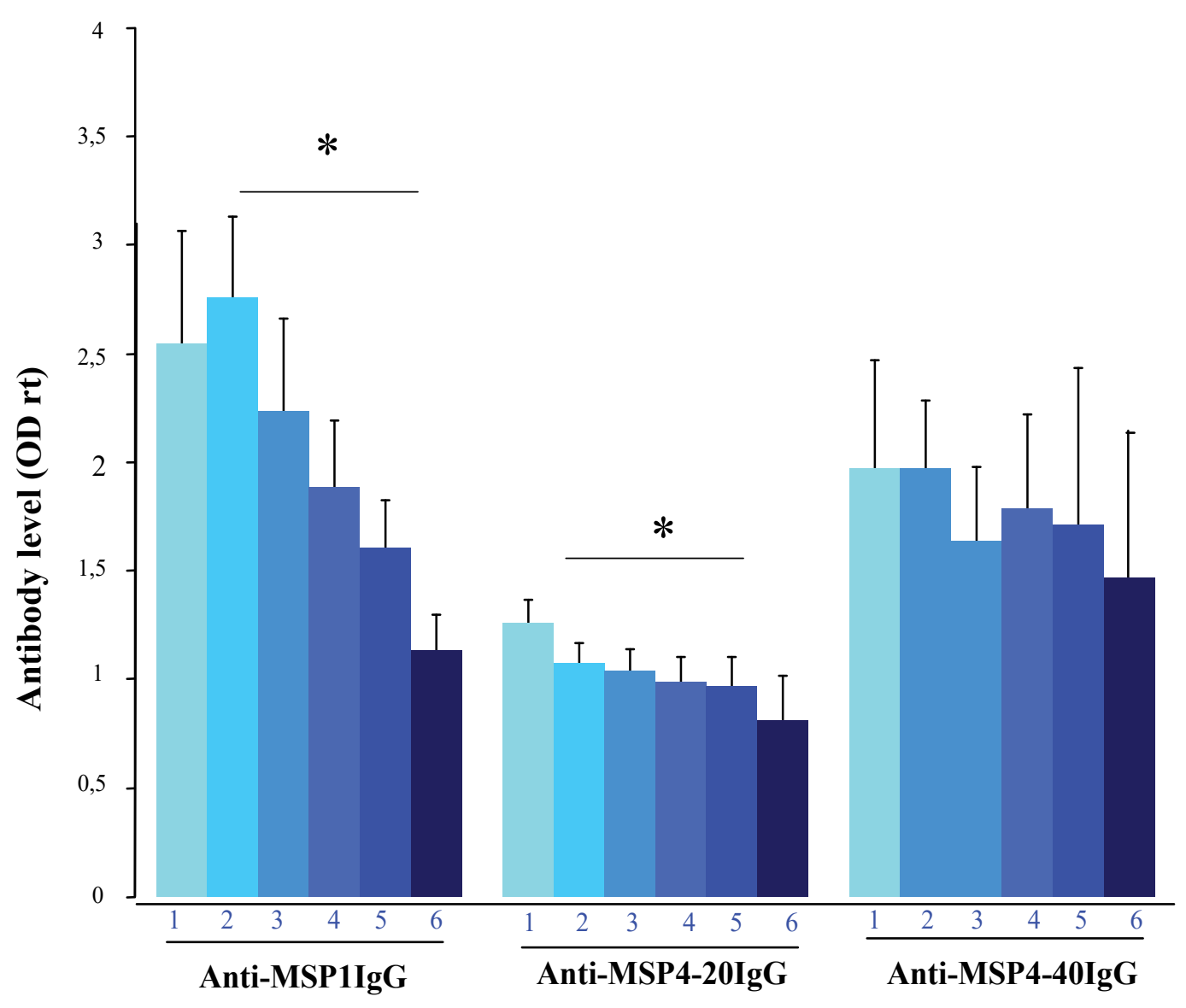

Blue figures: number of impaired organs

": Significant difference

Figure 4: Comparison of anti-MSP1, MSP4-20 and MSP4-40 lgG levels of patients hospitalized for severe malaria at the infectious and tropical department of the Fann university hospital, depending on the number of impaired organs.

and cytokine disorders causing immunopathological effects [10]. Thiaw $M$, in his study in Dakar, reported a positive correlation between the number of visceral failures per patient and serum concentrations of pro and anti-inflammatory cytokines (IL-6, TNF- $\alpha$, IL-10) [22]. Our results are perfectly complementary to these observations. The number of impaired organs per patient in our series increased with the fall in protective antibody levels (anti-MSP1 and MSP4-20 IgG). It is just the same phenomenon seen from the other angle of view.

The relationship between the outcome of severe malaria and the levels of antibodies against vaccine candidate antigens is no longer to be proven $[11,10]$. Mbengue, et al. [14] reported a negative correlation between hospital lethality of severe malaria and anti-GPI antibody levels, in 2015 and recently between cerebral malaria-related lethality and anti-MSP1p19 and MSP-3 antibody levels [11]. In our study, elevated anti-MSP4-40 IgG levels were statistically associated with low lethality. These antibody-dependent protective effects result from several mechanisms. Antibody-Dependent Cellular Inhibition $(A D C l)$ in vitro experiments demonstrated that cytophilic antibodies directed against the MSP3 and GLURP antigens (Glutamate - Rich Protein) could inhibit the invasion of the erythrocytes by merozoites or neutralizing circulating parasites in the presence of monocytes [23]. In Burkina Faso, Aucan, et al. [24] showed a negative association between anti-RESA (Ring-infected Erythrocyte Surface Antigen) and antiMSP2 antibody titer with low parasite loads suggesting parasitic suppression dependent on immunoglobulins. In addition, strong anti-staging blood IgG responses in the series by O'Flaherty $\mathrm{K}$, et al. [25] were associated with more therapeutic effects in patients on artemisinin derivative-based treatments. Thus, the high levels of anti-MSP4-40 IgG in our survivors and the systematic use of Artesunate may partly explain the high cure rate $(88.37 \%)$ observed in our study.

\section{Limitations}

This study has certain limitations. It did not address the IgG subclasses especially the cytophilic IgG1 and 3 , which would provide more clarity and precision. In addition, other isotypes such as IgM and IgA important in the anti-malaria response were not measured. As our results are not derived from a vaccine trial, they are not sufficient as formal evidence of antiparasitic protection in severe forms of malaria. 


\section{Conclusion}

The morbidity and mortality associated with severe malaria remain a concern in Dakar, Senegal. This crosssectional, prospective, and analytical study aimed to analyze the IgG responses against $P$. falciparum MSP1, MSP4-20 and MSP4-40 antigens during severe malaria in patients hospitalized in the infectious diseases department of Fann university hospital. As this is not a vaccine trial, our findings have just suggested the involvement of anti-MSP1, MSP4-20, and MSP4 antibodies in the anti-plasmodial response in severe malaria. Nevertheless, the level of such specific types of MSP IgG might serve as prognostic biomarkers to prevent deadly outcomes in patients with severe malaria.

\section{Conflict of Interest}

The authors have declared no conflict of interest and no other relationship or activities that could appear to have influenced this study.

\section{Funding Statement}

Authors have not received any financial support from any organization or individual.

\section{Acknowledgements}

The authors are grateful to the staff of the infectious and tropical diseases department of Fann university hospital and patients who consent to participate in this study. The authors also acknowledged the technical support received from the Immunology laboratory of Cheikh Anta Diop University.

\section{References}

1. WHO (2020) World malaria report 2020.

2. Bhatt S, Weiss DJ, Cameron E, Bisanzio D, Mappin B, et al. (2015) The effect of malaria control on Plasmodium falciparum in Africa between 2000 and 2015. Nature 526: 207-211.

3. Mensah-Brown HE, Abugri J, Asante KP, Dwomoh D, Dosoo D, et al. (2017) Assessing the impact of differences in malaria transmission intensity on clinical and hematological indices in children with malaria. Malar J 16: 96.

4. Nkumama IN, O'Meara WP, Osier FHA (2017) Changes in malaria epidemiology in Africa and new challenges for elimination. Trends Parasitol 33: 128-140.

5. WHO (2019) World malaria report 2019.

6. Programme National de Lutte contre le Paludisme (2019) Bulletin épidémiologique annuel 2019 du paludisme au Sénégal.

7. Fowkes FJI, Boeuf P, Beeson JG (2016) Immunity to malaria in an era of declining malaria transmission. Parasitology 143: 139-153.

8. Phillips MA, Burrows JN, Manyando C, van Huijsduijnen $\mathrm{RH}$, Van Voorhis WC, et al. (2017) Malaria. Nat Rev Dis Primers 3: 17050.

9. Brejt JA, Golightly LM (2019) Severe malaria: Update on pathophysiology and treatment. Curr Opin Infect Dis 32: 413-418.
10. Boudin C, Baudon D, Devoucoux R (1984) Immunologie du paludisme: les relations hôte-parasite dans l'infection paludéenne. Etudes Médicales 1984: 91-104.

11. Mbengue B, Fall MM, Varela ML, Loucoubar C, Joos C, et al. (2019) Analysis of antibody responses to selected Plasmodium falciparum merozoite surface antigens in mild and cerebral malaria and associations with clinical outcomes. Clin Exp Immunol 196: 86-96.

12. Perraut $R$, Varela ML, Joos $C$, Diouf $B$, Sokhna $C$, et al. (2017) Association of antibodies to Plasmodium falciparum merozoite surface protein-4 with protection against clinical malaria. Vaccine 35: 6720-6726.

13. Mbengue B, Sylla Niang M, Ndiaye Diallo R, Diop G, Thiam $A$, et al. (2015) Analyses des réponses IgG dirigées contre des antigènes candidats vaccins dans le paludisme urbain non aggravé à Dakar (Sénégal): Variations suivant l'âge et les densités parasitaires. Bull Soc Pathol Exot 108: 94-101.

14. Mbengue B, Niang B, Niang MS, Varela ML, Fall B, et al. (2015) Inflammatory cytokine and humoral responses to Plasmodium falciparum glycosylphosphatidylinositols correlates with malaria immunity and pathogenesis. Immun Inflamm Dis 4: 24-34.

15. WHO (2014) Severe malaria. Trop Med Int Health 19: 7-131.

16. Leon $P$, Gabriel $P$ (2016) Guide de thérapeutique Perlemuter 2017. (9 $9^{\text {th }}$ edn), Elsevier/Masson, 2406-2435.

17. Kwenti TE, Kukwah TA, Kwenti TDB, Nyassa BR, Dilonga $\mathrm{MH}$, et al. (2019) Comparative analysis of IgG and IgG subclasses against Plasmodium falciparum MSP-1 ${ }_{19}$ in children from five contrasting bioecological zones of Cameroon. Malar J 18: 16.

18. Okech BA, Corran P, Todd J, Agwang C, Riley EM, et al. (2020) Differences in anti-malarial antibody concentrations and specificities between male and female Ugandan children. Arch Immunol 2: 11-17.

19. Dobaño C, Rogerson SJ, Mackinnon MJ, Cavanagh DR, Taylor TE, et al. (2008) Differential antibody responses to Plasmodium falciparum merozoite proteins in Malawian children with severe malaria. J Infect Dis 197: 766-774.

20. Wyss K, Wångdahl A, Vesterlund M, Hammar U, Dashti S, et al. (2017) Obesity and diabetes as risk factors for severe Plasmodium falciparum Malaria: Results from a Swedish nationwide study. Clin Infect Dis 65: 949-958.

21. Diop SA, Attinsounon CA, Fortes-Deguenonvo L, Cisse Dialo VMP, Seydi M (2014) Therapeutic itinerary of severe malaria in adults admitted to a teaching hospital in Dakar, Senegal. J Infect Dev Ctries 8: 1353-1355.

22. Thiaw M (2017) Paludisme grave: relation entre le phénotype clinique et les taux des cytokines circulante. Thèse Méd, Dakar, $\mathrm{n}^{\circ} 172$.

23. Bouharoun-Tayoun $H$, Druilhe $P$ (2015) AntibodyDependent Cell-Mediated Inhibition (ADCI) of Plasmodium falciparum: One-and two-step ADCl Assays. Methods Mol Biol 1325: 131-144.

24. Aucan $C$, Traoré $Y$, Tall $F$, Nacro B, Traoré-Leroux $T$, et al. (2000) High immunoglobulin G2 (lgG2) and low lgG4 levels are associated with human resistance to Plasmodium falciparum malaria. Infect Immun 68: 1252-1258.

25. O'Flaherty K, Maguire J, Simpson JA, Fowkes FJI (2017) Immunity as a predictor of anti-malarial treatment failure: A systematic review. Malar J 16: 158. 